\title{
New results for algebraic tensor reduction of Feynman integrals
}

\section{Jochem Fleischer}

Fakultät für Physik, Universität Bielefeld, Universitätsstr. 25, 33615 Bielefeld, Germany

\section{Tord Riemann*}

Deutsches Elektronen-Synchrotron DESY, Platanenallee 6, 15738 Zeuthen, Germany

E-mail: Tord.Riemannedesy.de

\section{Valery Yundin}

Niels Bohr International Academy and Discovery Center, Niels Bohr Institute, University of Copenhagen, Blegdamsvej 17, DK-2100, Copenhagen, Denmark

\begin{abstract}
We report on some recent developments in algebraic tensor reduction of one-loop Feynman integrals. For 5-point functions, an efficient tensor reduction was worked out recently and is now available as numerical C++ package, PJFry, covering tensor ranks until five. It is free of inverse 5point Gram determinants and inverse small 4-point Gram determinants are treated by expansions in higher-dimensional 3-point functions. By exploiting sums over signed minors, weighted with scalar products of chords (or, equivalently, external momenta), extremely efficient expressions for tensor integrals contracted with external momenta were derived. The evaluation of 7-point functions is discussed. In the present approach one needs for the reductions a $(d+2)$-dimensional scalar 5-point function in addition to the usual scalar basis of 1- to 4-point functions in the generic dimension $d=4-2 \varepsilon$. When exploiting the four-dimensionality of the kinematics, this basis is sufficient. We indicate how the $(d+2)$-dimensional 5-point function can be evaluated.
\end{abstract}

10th International Symposium on Radiative Corrections (Applications of Quantum Field Theory to Phenomenology) - Radcor2011

September 26-30, 2011

Mamallapuram, India

\footnotetext{
* Speaker.
} 


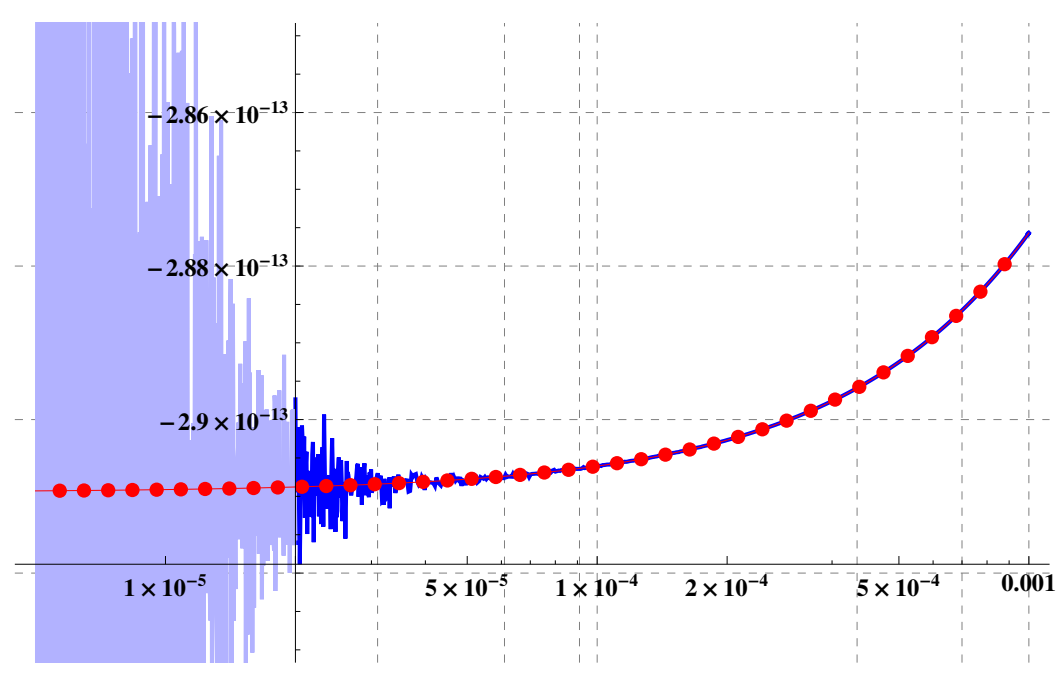

Figure 1: The 5-point tensor coefficient $E_{3333}$ in the region of a vanishing sub-Gram determinant. Blue curve: conventional Passarino-Veltman reduction [18], red curve: PJFry [14, 16, 17] . Figure copyright: V. Yundin, 2011.

\section{Introduction}

In recent years, we worked out a tensor reduction formalism for one-loop Feynman integrals with more than four legs. Tensor integrals are

$$
I_{n}^{\mu_{1} \cdots \mu_{R}}=\int \frac{d^{d} k}{i \pi^{d / 2}} \frac{\prod_{r=1}^{R} k^{\mu_{r}}}{\prod_{j=1}^{n} c_{j}},
$$

with denominators $c_{j}=\left(k-q_{j}\right)^{2}-m_{j}^{2}+i \varepsilon$ and chords $q_{j}$.

An efficient and stable numerical implementation is of high relevance for the description of multi-leg final states at the LHC. The state of the art has been regularly summarized at the "Les Houches Workshops on Physics at TeV Colliders", see e.g. [1] or the forthcoming proceedings of the 2012 edition. For a broader context, see also [2-4] and references therein.

The basics of our algebraic approach were formulated in [5-7]. In a series of papers, we performed the explicit reduction of 5- and 6-point tensors [8-15]. Arbitrary internal masses and external virtualities are allowed. Numerical singularities arising from inverse small 5-point Gram determinants are avoided and those from inverse small 4-point Gram determinants are safely evaluated by series of 3-point functions in higher dimensions with improvements by Padé approximants [14]. Based on that, the numerical C++ package PJFry was made available open-source $[14,16,17]$. An instructive example of typical output for a 6-point function is figure 1 ; for details on the kinematics see [17].

Since then, the interesting case of contractions of tensor integrals with external momenta was studied. After such contractions, the resulting scalar quantities are compact linear combinations of the basic scalar integrals with factorizing, simple combinations of signed minors and scalar products of external momenta [19, 20]. 
These developments were summarized at the conference, but because they were described already in earlier write-ups, we restrict ourselves here to the above quotations. Quite recently, we also studied the question of how to extend the tensor reductions to more than $n=6$ external legs. We again relied on the earlier treatment given in [7]. The reductions may be performed consistently in terms of 1- to 4-point scalar functions, as was done for $n \leq 6$, but one has in this framework as an additional element of the basis the 5-point function $I_{5}^{d+}$ in $d=6-2 \varepsilon$ dimensions.

This is described in section 2. The additional integral $I_{5}^{[d+]}$ is finite, but it is not contained in packages with scalar integrals like LoopTools/FF [21, 22], QCDLoop/FF [23, 22], or OneLOop [24]. In section 3, we indicate how to evaluate this integral directly.

After the conference we studied the approach due to [25, 26]. It allows to avoid the use of $I_{5}^{[d+]}$. This, together with the effects of contractions with external momenta, is studied for 7- and 8 -point tensor integrals in [27], but the details for higher tensor ranks have to be worked out yet.

\section{Tensor reduction for 7-point functions with recurrence relations}

Let us shortly repeat the reasoning for 6-point functions, and then switch to $n>6$. In [12] we have set up the tensor reduction for the 5 -point functions of rank $R$, expressing any $(5, R)$ pentagon by a $(5, R-1)$ pentagon plus $(4, R-1)$ boxes:

$$
I_{5}^{\mu_{1} \ldots \mu_{R-1} \mu}=I_{5}^{\mu_{1} \ldots \mu_{R-1}} Q_{0}^{\mu}-\sum_{s=1}^{5} I_{4}^{\mu_{1} \ldots \mu_{R-1}, s} Q_{s}^{\mu}
$$

In $[14]^{1}$ we have also given the corresponding homogeneous formula for 6-point functions

$$
I_{6}^{\mu_{1} \ldots \mu_{R-1} \mu}=-\sum_{s=1}^{6} I_{5}^{\mu_{1} \ldots \mu_{R-1}, s} Q_{s}^{0, \mu}
$$

with $p_{6}=0$ and

$$
Q_{s}^{0, \mu}=\sum_{i=1}^{5} q_{i}^{\mu} \frac{\left(\begin{array}{l}
50 \\
i 0
\end{array}\right)_{6}}{\left(\begin{array}{l}
0 \\
0
\end{array}\right)_{6}} .
$$

Now we follow [7]. For a reduction of 5-point functions two recursion relations are in principle sufficient:

$$
\begin{aligned}
v_{j} \mathbf{j}^{+} I_{n}^{(d+2)} & =\frac{1}{()_{n}}\left[-\left(\begin{array}{l}
j \\
0
\end{array}\right)_{n}+\sum_{k=1}^{n}\left(\begin{array}{l}
j \\
k
\end{array}\right)_{n} \mathbf{k}^{-}\right] I_{n}^{d}, \\
\left(d-\sum_{i=1}^{n} v_{i}+1\right) I_{n}^{(d+2)} & =\frac{1}{()_{n}}\left[\left(\begin{array}{l}
0 \\
0
\end{array}\right)_{n}-\sum_{k=1}^{n}\left(\begin{array}{l}
0 \\
k
\end{array}\right)_{n} \mathbf{k}^{-}\right] I_{n}^{d},
\end{aligned}
$$

where $\mathbf{i}^{ \pm}, \mathbf{j}^{ \pm}, \mathbf{k}^{ \pm}$act by shifting the indices $v_{i}, v_{j}, v_{k}$ by \pm 1 . They do not work out for 6-point functions since ()$_{n}=0$ for $n \geq 6$. A further recursion relation, which does not decrease dimension, reads

$$
v_{j} \mathbf{j}^{+} I_{n}^{(d)}=\frac{1}{\left(\begin{array}{l}
0 \\
0
\end{array}\right)_{n}} \sum_{k=1}^{n}\left(\begin{array}{c}
0 j \\
0 k
\end{array}\right)_{n}
$$

\footnotetext{
${ }^{1}$ See also [28].
} 
If reduction of dimension is acceptable, then the above double sum is often reduced by means of

$$
\sum_{j=1}^{n} v_{j} \mathbf{j}^{+} I_{n}^{(d+2)}=-I_{n}^{(d)}
$$

Further one uses (4.4) of [10] together with (4.5),

$$
\sum_{i=1}^{5} q_{i}^{\mu}\left(\begin{array}{l}
0 \\
i
\end{array}\right)_{6}=0
$$

Collecting the above, 6-point functions may be reduced.

For 7-point functions, things are a bit more involed because we have ()$_{7}=0$ and $\left(\begin{array}{l}0 \\ j\end{array}\right)_{7}=0$. Nevertheless one can apply recursion relations based on (2.6) and (2.7), see [7, 10]:

$$
\begin{aligned}
& \left(\begin{array}{l}
0 \\
0
\end{array}\right)_{n} I_{n, i}^{[d+]^{x}}=[n+1-(d+2 x)]\left(\begin{array}{l}
0 \\
i
\end{array}\right)_{n} I_{n}^{[d+]^{x}}+\sum_{r=1}^{n}\left(\begin{array}{c}
0 i \\
0 r
\end{array}\right)_{n} I_{n-1}^{[d+]^{(x-1), r}}, \\
& \left(\begin{array}{l}
0 \\
0
\end{array}\right)_{n} v_{i j} I_{n, i j}^{[d+]^{x}}=[n+2-(d+2 x)]\left(\begin{array}{l}
0 \\
j
\end{array}\right)_{n} I_{n, i}^{[d+]^{x}}+[i]^{j}+\sum_{r=1, r \neq i}^{n}\left(\begin{array}{c}
0 j \\
0 r
\end{array}\right)_{n} I_{n-1, i}^{[d+]^{(x-1), r}}, \\
& \left(\begin{array}{l}
0 \\
0
\end{array}\right)_{n} v_{i j k} I_{n, i j k}^{[d+]^{x}}=[n+3-(d+2 x)]\left(\begin{array}{l}
0 \\
k
\end{array}\right)_{n} I_{n, i j}^{[d+]^{x}}+[i j]_{r e d}^{k}+\sum_{r=1, r \neq i j}^{n}\left(\begin{array}{c}
0 k \\
0 r
\end{array}\right)_{n} I_{n-1, i j}^{[d+]^{(x-1), r}}, \\
& \left(\begin{array}{l}
0 \\
0
\end{array}\right)_{n} v_{i j k l} I_{n, i j k l}^{[d+]^{x}}=[n+4-(d+2 x)]\left(\begin{array}{l}
0 \\
l
\end{array}\right)_{n} I_{n, i j k}^{[d+]^{x}}+[i j k]_{r e d}^{l}+\sum_{r=1, r \neq i j k}^{n}\left(\begin{array}{c}
0 l \\
0 r
\end{array}\right)_{n} I_{n-1, i j k}^{[d+]^{(x-1), r}},
\end{aligned}
$$

where

$$
\begin{aligned}
{[i]^{j} } & =\left(\begin{array}{c}
0 j \\
0 i
\end{array}\right)_{n} I_{n}^{[d+]^{(x-1)}}, \\
{[i j]^{k} } & =\left(\begin{array}{c}
0 k \\
0 i
\end{array}\right)_{n} I_{n, j}^{[d+]^{(x-1)}}+\left(\begin{array}{c}
0 k \\
0 j
\end{array}\right)_{n} I_{n, i}^{[d+]^{(x-1)}},
\end{aligned}
$$

and the $[i j]_{\text {red }}^{k}$ is $[i j]^{k}$, but without repetition of equal indices $i, j$.

In [7] an idea of how to proceed for 7-point functions was formulated - details, however, were not given. For the 7-point vector one obtains from [5]

$$
I_{7}^{\mu}=-\sum_{i=1}^{7} q_{i}^{\mu} I_{7, i}^{[d+]}
$$

The (2.11) with $\left(\begin{array}{l}0 \\ 0\end{array}\right)_{7}=0$ and $\left(\begin{array}{l}0 \\ k\end{array}\right)_{7}=0$ yields for $i=j=k$ and $x=2$

$$
\left(\begin{array}{c}
0 i \\
0 i
\end{array}\right)_{7} I_{7, i}^{[d+]}+\sum_{r=1, r \neq i}^{7}\left(\begin{array}{c}
0 i \\
0 r
\end{array}\right)_{7} I_{6, i i}^{[d+], r}=0 .
$$

Since for the 6-point function ()$_{6} \equiv\left(\begin{array}{l}r \\ r\end{array}\right)_{7}=0$, we have from eq. (55) of [7]

$$
I_{6, i i}^{[d+], r}=\sum_{s=1, s \neq i}^{7} \frac{\left(\begin{array}{l}
R r \\
s r
\end{array}\right)_{7}}{\left(\begin{array}{l}
R r \\
0 r
\end{array}\right)_{7}} I_{5, i i}^{[d+], r s}+\frac{\left(\begin{array}{l}
R r \\
i r
\end{array}\right)_{7}}{\left(\begin{array}{l}
R r \\
0 r
\end{array}\right)_{7}} I_{6, i}^{[d+], r}, \quad R=\text { any value of } 0, \ldots, 7 .
$$


Applying in standard manner the recursion for the 5-point function

$$
\left.v_{i j} I_{5, i j}^{[d+]^{l}}=-\frac{\left(\begin{array}{l}
0 \\
j
\end{array}\right)_{5}}{()_{5}} I_{5, i}^{[d+]^{(l-1)}}+\sum_{s=1}^{5} \frac{\left(\begin{array}{l}
s \\
j
\end{array}\right)_{5}}{()_{5}} I_{4, i}^{[d+]}\right]^{(l-1), s}+\frac{\left(\begin{array}{l}
i \\
j
\end{array}\right)_{5}}{()_{5}} I_{5}^{[d+]^{[l-1)}}
$$

we replace $I_{5, i i}^{[d+], r s}$ by integrals of the type $I_{5, i}$ and $I_{4, i}^{s}$, the dimension of which must not be reduced. Therefore we have to apply recursion (2.6) in the form

$$
\left(\begin{array}{l}
0 \\
0
\end{array}\right)_{n} I_{n, j}=-[d-(n+1)]\left(\begin{array}{l}
0 \\
j
\end{array}\right)_{n} I_{n}-\sum_{i, k, i \neq k}\left(\begin{array}{l}
0 j \\
0 k
\end{array}\right)_{n} I_{n-1, i}^{k}, \quad n=5,4,3,2,
$$

i.e. starting at $n=2$ and increasing $n$ step by step, we obtain the desired integrals $I_{5, i}$ and $I_{4, i}^{S}$. For $n=2$ we have

$$
\left.\left(\begin{array}{l}
0 \\
0
\end{array}\right)_{2} I_{2, j}=-[d-3)\right]\left(\begin{array}{l}
0 \\
j
\end{array}\right)_{2} I_{2}-\left(\begin{array}{l}
0 j \\
02
\end{array}\right)_{2} I_{1,1}^{2}-\left(\begin{array}{l}
0 j \\
01
\end{array}\right)_{2} I_{1,2}^{1}
$$

with

$$
\begin{aligned}
& I_{1,1}^{2}=\frac{d-2}{2 m_{1}^{2}} I_{1}\left(m_{1}^{2}\right), \\
& I_{1,2}^{1}=\frac{d-2}{2 m_{2}^{2}} I_{1}\left(m_{2}^{2}\right) .
\end{aligned}
$$

The problematic case is the integral $I_{6, i}^{[d+], r}$ for which we can write similarly to (2.17)

$$
I_{6, i}^{[d+], r}=\sum_{s=1, s \neq i}^{7} \frac{\left(\begin{array}{l}
R r \\
s r
\end{array}\right)_{7}}{\left(\begin{array}{c}
R r \\
0 r
\end{array}\right)_{7}} I_{5, i}^{[d+], r s}+\frac{\left(\begin{array}{c}
R r \\
i r
\end{array}\right)_{7}}{\left(\begin{array}{l}
R r \\
0 r
\end{array}\right)_{7}} I_{6}^{[d+], r} .
$$

The $I_{6}^{[d+], r}$ cannot so easily be eliminated as in the case of the 6-point function, where the vanishing of (2.8) was used. Inserting (2.23) into (2.17), there occurs $\left(\begin{array}{c}R r \\ i r\end{array}\right)_{7}^{2}$, i.e. quadratic, so that the right hand side of (2.8) does not vanish.

\section{Numerical evaluation of higher-dimensional scalar integrals}

Since in the described approach the higher dimensional integral $I_{6}^{[d+], r}$ cannot be eliminated, it is needed to investigate the possibilty for its numerical evaluation - if one wants to continue with this approach. First of all one would reduce the 6-point function to 5-point functions by

$$
I_{6}^{[d+], r}=\sum_{s=1}^{7} \frac{\left(\begin{array}{l}
R r \\
s r
\end{array}\right)_{7}}{\left(\begin{array}{l}
R r \\
0 r
\end{array}\right)_{7}} I_{5}^{[d+], r s},
$$


such that the problem is shifted to the numerical evaluation of the $I_{5}^{[d+], r s}$. This, however, is a well known pathological case, since reducing the 6-dimensional 5-point function to 4-dimensional 5and 4-point functions, one has

$$
I_{5}^{[d+]}=\left[\frac{\left(\begin{array}{l}
0 \\
0
\end{array}\right)_{5}}{()_{5}} I_{5}-\sum_{s=1}^{5} \frac{\left(\begin{array}{l}
0 \\
s
\end{array}\right)_{5}}{()_{5}} I_{4}^{s}\right] \frac{1}{d-4},
$$

i.e. for $d=4$ one meets a division by zero, $\frac{0}{0}$. In [29], however, an interesting approach has been proposed, which can also be applied to handle this case. In fact, the idea is to go to even higher dimensions, which provides good numerical stability. ${ }^{2}$ One-loop $n$-point integrals in arbitrary dimension $d$ can be expressed in standard manner in terms of Feynman parameters as

$$
I_{n}^{(d)}=\Gamma\left(n-\frac{d}{2}\right) \int_{0}^{1} d x_{1} \ldots \int_{0}^{1} d x_{n-1} J_{n} h_{n}^{d / 2-n},
$$

where

$$
J_{n}=x_{n-2} x_{n-3}^{2} \cdots x_{1}^{n-2},
$$

and $h_{n}$ is a polynomial in the integration variables as well, containing also masses and momenta squared. The idea is to transform these integrals into integrals of higher dimension $D=d-2 \varepsilon+$ $2 n-2$. For small $\varepsilon$ the expansion of $I_{n}^{(d+2 n-2)}$ in $\varepsilon$ then reads [29]

$$
I_{n}^{(d+2 n-2)}=\Gamma(1+\varepsilon)\left[-\frac{s_{n}}{\varepsilon}-s_{n}-R_{n}+O(\varepsilon)\right],
$$

where $s_{n}$ can be written as

$$
s_{n}=\frac{1}{(n+1) !} \sum_{i, j=1}^{n} Y_{i j},
$$

with $Y_{i j}=-\left(q_{i}-q_{j}\right)^{2}+m_{i}^{2}+m_{j}^{2}$ and it is

$$
R_{n}=\int_{0}^{1} d x_{1} \ldots \int_{0}^{1} d x_{n-1} J_{n} h_{n} \ln \left(h_{n}\right)
$$

Obviously in such an integral no infinities occur anymore in the integrand and numerical integration is straightforward. It might even be possible and useful to evaluate it analytically. In our case of a 5 -point function we have $n=5$, i.e. this formula applies for the integral $I_{5}^{[d+]^{4}}$. Applying recursion relations, we can express (3.2) as

$$
\begin{aligned}
I_{5}^{[d+]}= & (d-2) d(d+2) \frac{()_{5}^{3}}{\left(\begin{array}{l}
0 \\
0
\end{array}\right)_{5}^{3}} I_{5}^{[d+]^{4}}+(d-2) d \frac{()_{5}^{2}}{\left(\begin{array}{l}
0 \\
0
\end{array}\right)_{5}^{3}} \sum_{s=1}^{5}\left(\begin{array}{l}
s \\
0
\end{array}\right) I_{4}^{[d+]^{3}, s} \\
& +(d-2) \frac{()_{5}}{\left(\begin{array}{l}
0 \\
0
\end{array}\right)_{5}^{2}} \sum_{s=1}^{5}\left(\begin{array}{l}
s \\
0
\end{array}\right) I_{4}^{[d+]^{2}, s}+\frac{1}{\left(\begin{array}{l}
0 \\
0
\end{array}\right)_{5}} \sum_{s=1}^{5}\left(\begin{array}{l}
s \\
0
\end{array}\right) I_{4}^{[d+], s} .
\end{aligned}
$$

Here we have now the desired representation of $I_{5}^{[d+]^{4}}$ in terms of higher-dimensional 4-point functions, for which standard recursions can be used to reduce them to integrals in generic dimension $d$. In this way 7-point functions could finally be dealt with.

\footnotetext{
${ }^{2}$ Other approaches to the numerical treatment of higher-dimensional scalar functions are e.g. [30, 31].
} 


\section{Acknowledgments}

The authors are grateful to G. Heinrich and S. Dittmaier for communication. TR would like to thank the Organizers of Radcor 2011, D. Indumathi, Prakash Mathews, Andreas Nyffeler, and V. Ravindran for their warm hospitality and a perfectly organized conference. This work is supported in part by Sonderforschungsbereich/Transregio SFB/TRR 9 of DFG "Computergestützte Theoretische Teilchenphysik" and by European Initial Training Network LHCPHENOnet PITNGA-2010-264564.

\section{References}

[1] J. Andersen et al., The SM and NLO Multileg Working Group: Summary report, arXiv: 1003.1241 . Proceedings of the 6th Workshop on Physics at TeV colliders, 8-26 June 2009, Les Houches, France. Dedicated to Thomas Binoth.

[2] J. Blümlein, S. Moch, and Tord Riemann (eds.), Loops and Legs in Quantum Field Theory, Nucl. Phys. B (Proc. Suppl.) 205-206 (2010) 1. Proceedings of the 10th DESY Workshop on Elementary Particle Theory, Wörlitz, Germany, April 25-30, 2010.

[3] T. Speer, F. Carminati, and M. Werlen (eds.), Advanced computing and analysis techniques in physics research, PoS(ACAT08). Proceedings of the 12th International Workshop ACAT, 3-7 November 2008, Erice, Italy.

[4] ETH Zürich (ed.), Applications of Quantum Field Theory to Phenomenology, PoS(RADCOR2009) (2010). Proc. of 9th International Symposium on Radiative Corrections, 25-30 October 2009, Ascona, Switzerland.

[5] A. I. Davydychev, A simple formula for reducing Feynman diagrams to scalar integrals, Phys. Lett. B263 (1991) 107-111. doi:10.1016/0370-2693(91)91715-8.

[6] O. Tarasov, Connection between Feynman integrals having different values of the space-time dimension, Phys.Rev. D54 (1996) 6479-6490, [hep-th/9606018].

[7] J. Fleischer, F. Jegerlehner, and O. Tarasov, Algebraic reduction of one-loop Feynman graph amplitudes, Nucl. Phys. B566 (2000) 423-440, [hep-ph/ 9907327$].$

[8] J. Fleischer. Application of Mellin-Barnes representation to the calculation of massive five-point functions in Bhabha scattering, talk held at Conference on Frontiers in Perturbative Quantum Field Theory, 14-16 June 2007, ZiF, Bielefeld, Germany, slides.

[9] T. Diakonidis, J. Fleischer, J. Gluza, K. Kajda, T. Riemann, and J. Tausk, On the tensor reduction of one-loop pentagons and hexagons, Nucl. Phys. Proc. Suppl. 183 (2008) 109-115, [arXiv:0807.2984].

[10] T. Diakonidis, J. Fleischer, J. Gluza, K. Kajda, T. Riemann, and J. Tausk, A complete reduction of one-loop tensor 5- and 6-point integrals, Phys. Rev. D80 (2009) 036003, [arXiv: 0812.2134 ].

[11] J. Gluza, K. Kajda, T. Riemann, and V. Yundin, New results for loop integrals: AMBRE, CSectors, hexagon, PoS(ACAT08) 124 (2009) [arXiv: 0902 . 4830].

[12] T. Diakonidis, J. Fleischer, T. Riemann, and J. B. Tausk, A recursive reduction of tensor Feynman integrals, Phys. Lett. B683 (2010) 69-74, [arXiv: 0907.2115$].$

[13] T. Diakonidis, J. Fleischer, T. Riemann, and B. Tausk, A recursive approach to the reduction of tensor Feynman integrals, PoS RADCOR2009 (2009) 033, [arXiv: 1002.052 9]. 
[14] J. Fleischer and T. Riemann, Complete algebraic reduction of one-loop tensor Feynman integrals, Phys. Rev. D83 (2011) 073004, [arXiv: 1009 . 4436].

[15] J. Fleischer and T. Riemann, Simplifying 5-point tensor reduction, Acta Phys. Polon. B42 (2011) 2371-2378, [arXiv:1111.4153].

[16] V. Yundin C++ package PJFry. Available at https://github.com/Vayu/PJFry.

[17] J. Fleischer, T. Riemann, and V. Yundin, PJFry - a C++ package for tensor reduction of one-loop Feynman integrals. Contrib. to Proc. of the Workshop "Physics at TeV Colliders", 30 May - 17 June 2011, Les Houches, France, preprint DESY 11-252 (2011).

[18] G. Passarino and M. Veltman, One loop corrections for $e^{+} e^{-}$annihilation into $\mu^{+} \mu^{-}$in the Weinberg model, Nucl. Phys. B160 (1979) 151.

[19] J. Fleischer and T. Riemann, Calculating contracted tensor Feynman integrals, Phys.Lett. B701 (2011) 646-653, [arXiv:1104.4067].

[20] J. Fleischer, T. Riemann, and V. Yundin, One-loop tensor Feynman integral reduction with signed minors, 1112.0500 . Talk held at ACAT 2011, to appear in JPCS.

[21] T. Hahn and M. Perez-Victoria, Automatized one-loop calculations in four and d dimensions, Comput. Phys. Commun. 118 (1999) 153, [hep-ph/9807565].

[22] G. J. van Oldenborgh, FF: A Package to evaluate one loop Feynman diagrams, Comput. Phys. Commun. 66 (1991) 1-15. doi:10.1016/0010-4655(91)90002-3, scanned version at http://ccdb3fs.kek.jp/cgi-bin/img_index?9004168.

[23] R. K. Ellis and G. Zanderighi, Scalar one-loop integrals for QCD, JHEP 02 (2008) 002, [arXiv:0712.1851].

[24] A. van Hameren, Oneloop: For the evaluation of one-loop scalar functions, Comput.Phys.Commun. 182 (2011) 2427-2438, [arXiv: 1007 .4716].

[25] Z. Bern, L. J. Dixon, and D. A. Kosower, Dimensionally Regulated One-Loop Integrals, Phys. Lett. B302 (1993) 299-308 [Erratum-ibid. B 318 (1993) 649], [hep-ph / 9212308 ].

[26] T. Binoth, J. Guillet, G. Heinrich, E. Pilon, and C. Schubert, An algebraic / numerical formalism for one-loop multi-leg amplitudes, JHEP 10 (2005) 015, [hep-ph / 0504267 ].

[27] J. Fleischer and T. Riemann, A solution for tensor reduction of one-loop n-point functions with $n \geq 6$, Physics Letters B $\mathbf{7 0 7}$ (2012) 375 - 380, [arXiv:1111.5821].

[28] A. Denner and S. Dittmaier, Reduction schemes for one-loop tensor integrals, Nucl. Phys. B734 (2006) 62-115, [hep-ph/0509141].

[29] F. Jegerlehner and O. Tarasov, FIRCLA, one-loop correction to $e^{+} e^{-} \rightarrow v \bar{v} H$ and basis of Feynman integrals in higher dimensions, Nucl. Phys. Proc. Suppl. 116 (2003) 83-87, [hep-ph/ 0212004 ].

[30] D. Bardin, L. Kalinovskaya, and F. Tkachov, New algebraic numeric methods for loop integrals: Some one loop experience, hep-ph/0012209.

[31] J. Fleischer, F. Jegerlehner, and O. V. Tarasov, A new hypergeometric representation of one-loop scalar integrals in d dimensions, Nucl. Phys. B672 (2003) 303-328, [hep-ph/ 0307113$].$ 